Teologia i Moralność, volumen 16(2021), numer 2(30)

doi: 10.14746/TIM.2021.30.2.6

ORCID: 0000-0002-3969-3954

JAROSŁAW WOJTKUN

Università Cattolica Giovanni Paolo II di Lublin

Facoltà di Teologia

\title{
Padre dei "figli prediletti di Dio" e Papa della famiglia - due maestri e annunciatori del Vangelo della vita
}

\section{Inroduzione}

Il monumento situato nel cortile dell'Università Cattolica Giovanni Paolo II di Lublino presenta due figure di spicco della Chiesa cattolica in Polonia: Giovanni Paolo II e il Primate del Millennio, il Cardinale Stefan Wyszyński, in un abbraccio fraterno, che il mondo ha visto durante la trasmissione della Santa Messa da S. Pietro per l'inaugurazione del pontificato il 22 ottobre 1978. I due grandi Pastori condividevano una comune responsabilità per la Chiesa in Polonia. Erano uniti dall'amicizia e dall'unità della trasmissione della verità del Vangelo. Giovanni Paolo II ha ripetutamente espresso grande rispetto e gratitudine al Primate Stefan Wyszyński. Già durante la prima udienza per i Polacchi dopo la sua elezione alla Sede di Pietro, si è rivolto al Primate Wyszyński con le parole seguenti: "Non ci sarebbe questo Papa polacco che oggi, pieno di timore di Dio, ma anche pieno di fiducia, inizia un nuovo pontificato, se non fosse la tua fede che non si ritrae davanti alla prigionia e alla sofferenza, se non fosse la tua eroica speranza, il tuo totale affidamento alla Madre della Chiesa" (Giovanni Paolo II 1978). Forse queste parole possono essere comprese non solo in contesto biografico, ma anche dottrinale. Gran parte del contenuto dell'insegnamento del Papa Polacco è coerente con ciò che predicò e scrisse prima il Cardinale Wyszyński. Per attraversare le parole del Papa, forse alcune di esse non sarebbero apparse nell'insegnamento di Giovanni Paolo II se non fossero state pronunciate prima dal grande Primate della 
Polonia, beato Stefan Wyszyński, la cui beatificazione era prevista per il centenario della nascita di Karol Wojtyła, per motivi epidemici ritardata di un anno.

Uno dei temi importanti discussi in precedenza dal Primate e che è apparso anche nell'insegnamento del Papa Polacco è stato il tema della vita. In questo studio vogliamo mostrare i punti di tangenza nel modo di affrontare il tema della vita, e arrivare anche a quei contenuti nel pensiero del Cardinale Wyszyński, che, anni dopo, avrebbero potuto essere fonte di ispirazione per il futuro Papa Karol Wojtyła.

\section{La vita personale come valore}

Uno dei temi centrali dell'insegnamento di Giovanni Paolo II era la questione del valore della vita e del suo carattere inviolabile. Il titolo stesso dell'enciclica sul Vangelo della vita sottolinea il valore e l'inviolabilità della vita umana: "Evangelium vitae sul valore e l'inviolabilità della vita umana". Il Papa sviluppa questa idea nell'introduzione al documento: "La presente Enciclica, frutto della collaborazione dell'Episcopato di ogni Paese del mondo, vuole essere dunque una riaffermazione precisa e ferma del valore della vita umana e della sua inviolabilità" (Giovanni Paolo II 1995, 5). Questo principio copre tutte le fasi della vita umana, dal concepimento alla morte naturale. E una regola senza eccezioni e senza concessioni. Appartiene all'essenza stessa della missione di Gesù Cristo: “Gesù, presentando l'essenza della sua missione redentrice, dice: «Io sono venuto perché le pecore abbiano la vita e l'abbiano in abbondanza (Gv 10,10)»" (Giovanni Paolo II 1995, 1). Questa affermazione sarà ripetuta altre quattro volte nell'enciclica. Il termine "in abbondanza" è degno di attenzione. Il significato del concetto di "vita" appare nella dimensione della pienezza, dell'abbondanza. Non sembra che il papa si riferisca solo a un'abbondanza nel senso soprannaturale. Il senso della vita non si limita alla sua più alta attualizzazione nella dimensione soprannaturale della comunione con Dio, ma abbraccia tutti i suoi aspetti e ogni singola dimensione. Questa convinzione è stata espressa più volte dal Papa Polacco. Durante un discorso all'aeroporto di Vienna, il 23 giugno 1988, ha lanciato un appello al superamento della cultura della morte ("Kultur des Todes") attraverso un deciso "sì" alla vita ("Ja zum Leben"). Poi ha aggiunto: "Si alla vita in tutte le sue fasi e forme". Allo stesso modo, a Salisburgo ha sottolineato questa totalità del valore della vita: il "sì" alla vita deve essere esteso a tutte le dimensioni della vita umana. Questo ampio raggio di comprensione della concezione evangelica della vita si ritrova anche nella parola rivolta ai vescovi tedeschi in visita Ad limina apostolorum, 19 dicembre 1992: "L'affermazione escatologica di Gesù che è venuto perché le sue pecore abbiano la vita e l'abbiano in 
abbondanza si applica indubbiamente ad ogni forma di vita" (Insegnamenti di Giovanni Paolo II, 1993).

Questa rassegna breve dei discorsi papali mostra come Giovanni Paolo II comprende il contenuto della vita e il suo carattere sacro. La natura sacra della vita umana implica il suo carattere inviolabile. Il pilastro dell'argomento teologico per la natura inviolabile della vita umana è la natura indiscutibile del comandamento "Non uccidere". La natura stessa dei comandamenti del Decalogo, compresi quelli formulati sotto forma di divieti, non ammette eccezioni. I precetti negativi "sono universalmente validi: essi obbligano tutti e ciascuno, sempre e in ogni circostanza" (Giovanni Paolo II 1993, 52). Il quinto comandamento del Decalogo non fa eccezione.

\subsection{Il valore della vita fisica}

Il principio della santità della vita umana si applica a tutte le sue dimensioni, non solo a quella soprannaturale, ma anche a quella temporale. Basta la luce della ragione naturale per percepirla e rispettarla. Intendiamo qua sia le componenti spirituali che fisiche di un essere umano. Il giusto posto accordato all'aspetto corporeo della natura dell'uomo non deve portare a nessuna manifestazione di dualismo. "La corporeità umana va presa in seria considerazione. Non è corretto dire che l'uomo ha un corpo. Bisogna roconoscere che l'uomo essenzialmente è corpo, e che senza corpo non c'è l'uomo. Il corpo può essere un limite per l'uomo, ma è innanzitutto costitutivo e perficiente" (Lobato 2003, 438). In tal modo Karol Wojtyła ha inteso il ruolo della componente corporea nella persona umana. Il corpo non è solo un elemento della natura umana, ma imprime anche ogni aspetto del funzionamento e della comunicazione, in altre parole, ogni aspetto della vita umana. "Wojtyła non accetta però neppure la visione secondo cui i fini propri della natura umana e tutto ciò che ha a che fare con l'appartenenza del corpo all'uno o altro sesso costituiscano soltanto la materia della libertà e non veicolino in se stessi nessun significato autenticamente personale. Nell'antropologia di Wojtyła il corpo insieme con la sua appartenenza sessuale fa parte dell'identità della persona" (Merecki 2019, 433).

La convinzione del valore fondamentale della vita anche nella sua dimensione terrena è giustificata da Giovanni Paolo II con riferimenti alla Rivelazione biblica. Già durante il suo viaggio verso la Terra Promessa, Israele ha scoperto quanto sia preziosa la sua vita agli occhi di Dio. Il popolo d'Israele ha scoperto gradualmente che "la sua vita non si trova alla merce di un faraone che può usarne con dispotico arbitrio; al contrario, essa è l'oggetto di un tenero e forte amore da parte di Dio" (Giovanni Paolo II 1995, 31). 
Anche per il Primate della Polonia beato Stefan Wyszyński, che ricordava ancora le atrocità della guerra, era di grande importanza sottolineare il valore della vita nella sua dimensione terrena. Il valore della vita terrena è così grande che l'uso corretto del tempo terreno può essere ancora più difficile dell'arte di morire. Nelle sue riflessioni scritte nell'ottobre 1952, poco prima della sua prigionia da parte del governo comunista, il Primate Wyszyński scriveva: "Mi sembra che l'ideale per oggi debba essere la capacità di vivere per la Chiesa in Polonia piuttosto che la capacità di morire. [...] Preferisco i miei sacerdoti all'altare, nell'ambone e nel confessionale che in una prigione" (Wyszyński 2017, 374). Poche settimane dopo, nei suoi appunti, aggiungeva: "Il fatto che sappiamo soffrire è stato dimostrato durante l'occupazione nazista. Ora dobbiamo dimostrare la capacità di lavorare" (Wyszyński 2017, 427).

La dottrina del valore della vita e della sua inviolabilità fin dal concepimento è stata definitivamente e solennemente confermata da Giovanni Paolo II, tanto per soddisfare pressupposti per l'insegnamento infallibile (cfr. Wojtkun 1998). Non è quindi una novità nell'insegnamento dei Pastori della Chiesa. Molto prima l'ha fatto, tra gli altri, il Primate Wyszyński. Il suo appello al rispetto del valore della vita aveva un contesto speciale legato alla situazione politica della Polonia in tempo comunista.

\subsection{Il fenomeno dell'aborto}

La vita umana appartiene alle realtà create. L'ovvietà di questa affermazione non richiede giustificazione. Tuttavia, è questa verità fondamentale che viene oggi ignorata in vari modi. Questo finisce nel trascurare e persino nel mettere in discussione la verità sulla natura sacra della vita umana. Ciò accade quando si vuole farne un oggetto di propria decisione, una realtà di cui si può disporre liberamente. Questo vale per la mia vita così come per quella degli altri. Il fenomeno dell'aborto è una manifestazione drammatica di tale trattamento della vita. Dopo aver riconquistato la libertà dalla dittatura comunista, le parole pronunciate da Giovanni Paolo II durante l'omelia a Kalisz nell'anno 1997 hanno aquistato un significato simbolico: "Ripeto tante volte - e ne sono certo - che il più grande pericolo che minaccia la pace oggi è un aborto. Se a una madre è permesso uccidere il proprio figlio, cosa può impedire a me e te di ucciderci a vicenda" (Giovanni Paolo II 1997, 35)? Quarant'anni prima, il beato Primate Wyszyński parlava con un tono altrettanto categorico dei pericoli dell'aborto: "Se il bambino più innocente e indifeso non può sentirsi al sicuro in una società, allora nessuno può sentirsi al sicuro in essa" (KAI 2020)! La Conferenza Episcopale Polacca, sotto la presidenza del Cardinale Wyszyński, l'ha dedicata nell'anno 1965 intera lettera ai sacerdoti al tema della difesa del- 
la vita concepita. La lettera si compone secondo tre pilastri: diagnosi del male, ricerca delle cause, modalità di superare (Listy Pasterskie 2003, 470-481).

L'aborto non è solo un peccato che colpisce la madre, il padre del bambino e gli esecutori dell'aborto. L'atteggiamento verso la vita concepita determina il futuro dell'intera nazione. Non c'è qui spazio per le sfumature, l'alternativa al rifiuto della natura inviolabile della vita umana concepita è, secondo Giovanni Paolo II, ovvia: "Una nazione che uccide i propri figli è una nazione senza futuro" (Giovanni Paolo II 1997, 35). Mentre l'avvertimento papale riguarda il futuro, Stefan Wyszyński colloca i pericoli dell'aborto nel presente. Si traducono proprio adesso in un quadro drammatico delle relazioni sociali, familiari e personali: "Se non sapranno (sostenitori dell'aborto) rispettare la vita piccolissima che nasce nella cellula della vita domestica, non rispetteranno la vite dei cittadini, perché impareranno a uccidere già nelle famiglie. In questo modo, invece di una comunità che dà la vita, si svilupperà una comunità di assassini. Sarà una nazione suicida [...]. Una tale nazione scomparirà" (KAI 2020).

Una peculiare "doppia voce", seppur rimandata nel tempo, riguarda anche la questione della legge che legalizza l'aborto. Stefan Wyszyński, di fronte alla legge che ammette il diritto all'aborto su richiesta introdotto nell'anno 1956 dal governo comunista, ha fatto appello al rispetto incondizionato della vita concepita. Sosteneva che la legalizzazione dell'aborto fosse assolutamente illegale. "Ogni cosiddetta legge che dà a chiunque l'opportunità di disporre e determinare la vita umana è illegalità. Ogni atto che sia finalizzato alla distruzione e all'abolizione del diritto umano alla vita è un reato" (KAI 2020). Le tendenze che portano alla liberalizzazione della legge sull'aborto in Polonia non appartengono al passato. Così, a distanza di anni, era necessario un intervento da parte del Papa. Giovanni Paolo II ha considerato come illegale una legge che ammette l'aborto con una serie di domande espresse durante l'omelia pronunciata a Radom il 4 giugno 1991: "Esiste una tale autorità umana, o esiste un parlamento che ha il diritto di legalizzare l'omicidio di un essere umano innocente e indifeso? Chi ha il diritto di dire: «È permesso uccidere», anche: «Dobbiamo uccidere», la dove è necessario proteggere e aiutare di più la vita" (Giovanni Paolo II 1991)?

Il carattere sacro e inviolabile della vita umana trae la sua giustificazione dalla verità sulla dignità dell'uomo. L'uomo è un essere che Dio ha voluto per sé stesso, e la vita umana, come atto speciale di creazione, dipende da Dio nella sua interezza. La sua portata tocca gli inizi, attraverso la sua storia, fino alla morte naturale. "L'amore di Dio non fa differenza tra un essere appena concepito mentre è ancora nel grembo materno e un bambino, un adolescente, una persona matura o un anziano. Non fa differenza perché in ognuno vede il suo riflesso e la sua somiglianza. Egli non fa differenza, perché in tutto riconosce il volto del suo Figlio unigenito, nel quale «ci ha scelti prima della fondazio- 
ne del mondo, $[\ldots]$ ci ha destinati ad essere suoi figli adottivi [...] secondo al decreto della sua volontà» (Ef 1,4-5). Questo amore sconfinato e quasi incomprensibile di Dio per l'uomo mostra quanto l'uomo stesso sia degno di amore, indipendentemente da altre ragioni - indipendentemente dalla sua intelligenza, bellezza, salute, giovinezza, e simili" (Benedetto XVI 2006, 55). L'amore di Dio per l'uomo, che "non fa differenza, perché vede in ciascuno il riflesso della sua immagine e somiglianza", si rivela nella grazia della vita di Dio donata all'uomo.

\section{La grazia di Dio dona la bellezza alla vita umana}

La vita divina nell'uomo è profondamente connessa con la vita nella dimensione naturale. Beato Stefan Wyszyński ha sintetizzato nei suoi appunti una delle sue prediche ai giovani come "rappresentazione di Dio che vive nel nostro essere umano". Il segno di questa vita è la chiamata a Dio che scaturisce da ogni sfera dell'esistenza umana: la mente, il cuore e anche il corpo. Dobbiamo usare il nostro corpo e la nostra anima per confessare Dio con la nostra bocca, con le nostre mani e con il nostro cuore (Wyszyński 2017, 44).

\subsection{La sensibilità estetica}

L'uso dell'anima come elemento spirituale dell'uomo si manifesta, tra l'altro, nella sensibilità estetica. Giovanni Paolo II tratta la sensibilità alla bellezza come un'emanazione della legge naturale nella natura umana. L'espressione di questa legge prende la forma di un imperativo: bisognia contemplare la bellezza (Giovanni Paolo II 1993, 51). La bellezza, organicamente connessa con i prodotti artistici dell'arte, dovrebbe sempre andare di pari passo con la bellezza interiore dell'uomo. Tale bellezza ha ispirato l'opera di beato Fra Angelico, che "si sentì profondamente chiamato dal paragone di Cristo di un buon albero e di un buon frutto a una duplice creatività: creò opere come creò se stesso" (Giovanni Paolo II 1986, 255). La bellezza della creatività artistica consiste nel cercare la giusta proporzione tra la bellezza delle opere e la bellezza dell'anima. Tanto importante quanto il bene, la bellezza e la verità come i componenti della cultura che plasmano l'uomo è l'amore, definito da Giovanni Paolo II come "il bisogno primordiale di tutta la cultura umana" (Giovanni Paolo II 2007, 775). Una cultura fondata sull'amore inteso come dono è capace di creare una communio personarum, cioè la cultura dell'amore, la civiltà dell'amore. Si vede qui lo specchio delle parole dell'insegnamento del Concilio Vaticano II, che hanno ispirato l'intero pontificato del Papa Polacco: „L'uomo, il quale in 
terra è la sola creatura che Iddio abbia voluto per se stesso, non possa ritrovarsi pienamente se non attraverso un dono sincero di sé" (Concilio Vaticano II 1965a, 24). L'amore nell'esercitare le opere della culura costituisce quindi un fattore più significatovo che crea e svilluppa persona umana (Rodziński 1998, 201-214).

La sensibilità alla bellezza dell'animo umano è uno dei fattori importanti che caratterizzano la figura del Stefan Wyszyński. I suoi appunti personali, redatti sistematicamente quasi ogni giorno, testimoniano la sua grande sensibilità per la bellezza dell'uomo, la bellezza del creato, la bellezza del lavoro umano. Lui ha potuto vedere nel lavoro quotidiano dei fratelli religiosi alla costruzione del tempio di Niepokalanów un'espressione di bellezza manifestata nell'amore e nel servizio. La solidità e l'integrità dell'opera che manifesta la pace francescana è persino seducente: "I frati che lavorano prestano poca attenzione ai visitatori. Sono impegnati con il loro lavoro amato. Gli sono devoti con edificante modestia, senza quel chiacchiericcio che abbonda sui giornali dove tutto è al di sopra della norma. Tutto qui è semplice, composto, modesto, ma davvero perbene e onesto. [...] Così lavora un lavoratore, animato dall'amore per il lavoro che serve" (Wyszyński 2017, 153).

\subsection{Il ruolo della grazia per la bellezza dell'uomo}

La grazia di Dio è un segno tangibile della bellezza umana. La grazia rende bello l'uomo e la bellezza dona alla grazia che è invisibile qualcosa di "tangibile". Nelle riflessioni del Primate Wyszyński si possono trovare tracce di una specifica traccia "empirica" della vita di Dio nell'uomo. Osservando i volti dei fedeli, Cardinale Wyszyński giunge alla conclusione che l'effetto "tangibile" della grazia santificante di Dio nelle persone è la gioia del loro sguardo e l'allegria delle anime (Wyszyński 2017, 337). Il Primate, con sorprendente semplicità, e anche con un pizzico di umorismo, ha saputo illustrare il meccanismo della grazia invisibile di Dio nell'uomo. Si ispirò al modo in cui i parrocchiani sono stati caratterizzati dal parroco di una delle parrocchie marittime. Lui ha usato il paragone per pescare. Tra i fedeli ci sono persone simili a "preziose lucioperca", ma ci sono anche "merluzzi sciocchi", "lucci testardi", "anguille mobili" e persino squali. Interrogato dal parroco, come raccogliere e integrare tutto questo, il Primate ha risposto: "Ogni pesce può essere preparato in modo che diventi gustoso, da poter essere mangiato, e che dire di un uomo, quando è condito dalla grazia di Dio e dallo zelo pastorale" (Wyszyński 2017, 337).

Dal punto di vista teologico, la verità sulla reciproca compenetrazione delle dimensioni terrena e soprannaturale della vita non era facile. Stefan Wyszyński ha potuto spiegare riferendosi ad un'altra osservazione della natu- 
ra. Da acuto osservatore della natura, usò la sua intuizione per illustrare verità di natura teologica. Il miracolo dell'amore di Dio è visibile, per esempio, nel mondo degli uccelli. Ha incoraggiato i suoi sacerdoti ad osservare il comportamento dei pulcini nei nidi: "Vediamo come il novellino segue ogni mossa della sua mamma alata. E tutto, che sia un bambino o un pulcino, chiede cibo con il suo piagnucolio. La vita soprannaturale, per volontà del Padre celeste, non è più povera di cuore della vita della natura. Il cristianesimo, così paterno, è anche materno, e solo allora potrà essere - per volontà di Dio - fecondo" (Wyszyński 2019a, 54).

Questa fecondità, che è l'effetto della grazia di Dio nell'uomo, è un elemento dell'identità cristiana. Beato Stefan Wyszyński ha voluto trasmettere ai sacerdoti la convinzione della necessità della grazia santificante per la conservazione dell'identità cristiana, preparandoli alla celebrazione del Millenium del battesimo della Polonia, celebrato nell'anno 1966. Vivere una vita cristiana e vivere nella grazia santificante sono realtà che funzionano sempre insieme. Tutte le manifestazioni della loro separazione furono chiamate dal Primate "la laicità del cattolicesimo nazionale". La perspicacia profetica del Primate può stupire. Alle Sante Messe celebrate dal cardinale Wyszyński hanno partecipato in quel tempo folle di fedeli. Lo stesso Primate lo ha sottolineato molte volte nelle note commemorative Pro memoria che scriveva quasi ogni giorno. In queste note troviamo l'espressioni come: "chiese piene di fedeli" (Wyszyński 2019a, 3), "enorme folla di credenti" (Wyszyński 2019a, 74), "folla straordinaria in chiesa" (Wyszyński 2019a, 154). Eppure, sotto la superficie di questa religiosità popolare della gente che afollava le chiese, lui vedeva insinuarsi il pericolo della secolarizzazione, in cui "tutto all'esterno è «cattolico» nelle parole e nei gesti, e solo nei cuori e nelle menti è tutto l'inferno di Dante. I cattolici polacchi devono essere convinti che la forza della nostra Chiesa dipende dalla forza spirituale dei fedeli che vivono nella grazia santificante. [...] L'anemia della vita morale non è colpa della Chiesa, ma dei cattolici deboli che amano la Chiesa e ad essa sono attaccati, ma non la ascoltano" (Wyszyński 2007, 121-122).

La minaccia della secolarizzazione di una società di consolidata tradizione cattolica era stata percepita dal Primate Wyszyński tre decenni prima dell'appello alla nuova evangelizzazione formulato da Giovanni Paolo II, come risposta al fenomeno sempre più diffuso di "secolarizzazione del cattolicesimo": "Si tratta, in particolare, dei paesi e delle nazioni del cosiddetto Primo Mondo, nel quale il benessere economico e il consumismo, anche se frammisti a paurose situazioni di povertà e di miseria, ispirano e sostengono una vita vissuta «come se Dio non esistesse». Ora l'indifferenza religiosa e la totale insignificanza pratica di Dio per i problemi anche gravi della vita non sono meno preoccupanti ed eversivi rispetto all'ateismo dichiarato" (Giovanni Paolo II 
1988, 34). La visione cristiana delle sfide legate al fenomeno della progressiva secolarizzazione e della "cultura della morte" non deve significare pessimismo e negatività. È "una preoccupazione positiva per la costruzione di una nuova cultura della vita come elemento essenziale della nuova evangelizzazione" (Nagórny 1997, 155).

\section{La paternità spirituale e i "padri della culla"}

La fecondità operata dalla grazia di Dio nell'uomo è sempre connessa con la paternità. Wyszyński ha mostrato in modo chiaro la compenetrazione delle dimensioni fisica e spirituale della paternità. Ciò avviene nell'interconnessione del sacramento del sacerdozio e del matrimonio. Questi due sacramenti si incontrano nel contesto di paternità alla cerimonia del matrimonio. Il Cardinale Wyszyński ha più volte paragonato la paternità fisica degli sposi con la paternità spirituale dei sacerdoti. La paternità dei sacerdoti è spirituale, la paternità fisica appartiene ai "padri della culla". Entrambi sono vicini l'uno all'altro. Già durante il sacramento del matrimonio, "i futuri padri di famiglia stanno agli altari del Signore davanti ai testimoni autentici della Chiesa - i padri spirituali - e si stringono la mano. Lì tre mani si incontrano: la mano della sposa nella mano del marito e la mano del sacerdote, che abbraccia queste due mani come se volesse trasmettere loro tutto lo spirito di Dio, affinché d'ora in poi tutto ciò che avvenga in loro possa veramente nascere da Dio" (Wyszyński 2021, 131). Il Primate Stefan Wyszyński ha sperimentato personalmente il potere vivificante della paternità di Dio. Guarito da una grave malattia, il 6 novembre 1977 ha incontrato i fedeli nella Basilica Cattedrale di Varsavia. Ringraziando per le preghiere per la sua intenzione, ha aggiunto: "Ciascuno di noi è frutto della volontà del Padre e dell'amore del Padre. [...] E poiché Dio è Amore, allora tutta la nostra vita è per noi ricca nell'amore. Ripeto spesso questa giustapposizione: la gente dice: «il tempo è denaro». Dico diversamente: «il tempo è amore». Il denaro è insignificante e l'amore dura" (Wyszyński 2007, 34).

\subsection{La fecondità spirituale e fisica}

La fecondità, che si traduce nell'impiantare la vita di Dio, si realizza anche a livello dei compiti educativi dei genitori. I genitori educano i figli alla fede. Tuttavia, l'attuazione di questi compiti può essere accompagnata dalla dolorosa esperienza del fallimento. Molti genitori sperimentano la realtà dei loro figli adolescenti che si allontanano dalle pratiche religiose. L'integrità religiosa della famiglia può essere sconvolta. L'iniziale avversione dei ragazzi 
per le pratiche religiose porta spesso al loro completo abbandono. I genitori che vivono tali situazioni sono pronti ad attribuire questo stato a errori educativi, sono accompagnati da un senso di colpa. Sono scoraggiati e rassegnati. Si rassegnano al processo di secolarizzazione dei propri figli, che sembra loro inevitabile e irreversibile. L'unità familiare soffre, l'armonia è turbata, l'amore è ferito, si verifica una crisi di identità genitoriale (cfr. Gatti 1991, 325; Wojtkun 2020, 80-89).

Tali situazioni provocano una riflessione più profonda sul meccanismo di trasmissione della vita di fede ai figlii. Non si tratta di trasmettere la fede come tradizione culturale. La trasmissione della fede non avviene per via ereditaria. L'"eredità" dei valori religiosi avviene solo in senso analogo, e nella misura consentita dal meccanismo di trasferimento del deposito culturale e morale della famiglia. Certo, il clima religioso in casa è di grande importanza, ma spesso non basta. Wyszyński fornisce preziosi suggerimenti in questo settore dei compiti dei genitori. Ha descritto il messaggio di fede come un messaggio speciale. Come i sacerdoti sono inviati a tutto il Popolo di Dio, così le madri e i padri sono inviati dalla Chiesa ai loro figli per predicare loro Dio. Prima ancora che i bambini capiscano i sacerdoti, capiscono già la lingua delle madri che raddrizzano le mani dei loro figli al segno della croce. I genitori sono responsabili di quanto Dio viene preso dai loro figli dalla famiglia, dalla scuola, dalla vita e dall'istruzione pubblica. Il primo posto nel condurre i figli a Dio spetta alla famiglia: "La famiglia è il centro più importante della santità" (Wyszyński 2021, 144). I compiti più urgenti della famiglia vertono sulle seguenti dimensioni della vita: "Creare un fronte di famiglie a difesa della vita, generare generazioni nel mondo per il bene della nazione e la gloria di Dio, cooperare con la benedizione di Dio, educare le giovani generazioni ad essere fedeli alla volontà di Dio di vivere, ad essere grate per la vita donata, a suscitare il rispetto per la vita, adoperarsi per una vita eterna felice" (Wyszyński 2021, 147).

Lo spazio dei compiti genitoriali così definito non consiste solo al loro trasferimento. La paternità e la genitorialità non solo donano la vita, ma ne sono la custode. In primo luogo, le madri devono essere custodi della vita. Sono "le ancelle del Padre di tutta la vita". Devono essere fedeli alla vita, soddisfacendo così la chiamata a trasmettere la vita ricevuta dal Padre celeste (cfr. Wyszyński 2020, 271). Padri e sacerdoti devono svolgere anche il compito di custodi della vita. La paternità fisica consiste nell'essere custode della culla. I sacerdoti, invece, sono i custodi dell'altare, del luogo dove Dio nasce e si comunica nell'uomo. Essere un custode dà luogo a responsabilità specifiche. Dovrebbero riunirsi in un'area di lavoro comune. Questa comunità di compiti è la fonte dell'appello del Primate della Polonia: "Seminate insieme la forza del corpo e dello spirito nella pianura polacca, perché la terra polacca sia sog- 
getta a Dio, che la terra di Polonia non sarebbe senz'acqua, deserta e selvaggia; che sia piena del popolo di Dio" (Wyszyński 2021, 132). Wyszyński non ha esitato a usare su questo argomento parole grandi: si tratta della ragion di Stato. La reciproca compenetrazione della paternità spirituale e della paternità delle culle è l'unica via di sopravvivenza della famiglia, è il nazionale "essere o non essere".

Poco più di vent'anni dopo, Giovanni Paolo II esprimerà un'idea simile. Alla famiglia avrà applicato le parole ammonitrici dei Padri del Concilio Vaticano II: 'L'epoca nostra, più ancora che i secoli passati, ha bisogno di questa sapienza, perché diventino più umane tutte le sue nuove scoperte. E' in pericolo, di fatto, il futuro del mondo, a meno che non vengano suscitati uomini più saggi" (Giovanni Paolo II 1981, 8). La saggezza dovrebbe andare di pari passo con la gratitudine espressa, tra l'altro, in gesti rispettosi e umili. Per il beato Stefan Wyszyński, questo gesto consiste nell'appoggiarsi alle mani di sua madre e di suo padre. Spiega questo gesto con un'espressione talmente calda e calorosa: "Le mani della madre e del padre hanno elaborato e servito un boccone di pane che viene da Dio e per il quale ogni giorno gridiamo a lui. E Dio ce lo darà da madre e padre. Ricordiamo con gratitudine i loro sforzi, il sacrificio della loro madre, che per molte notti ha vegliato sulla culla dei suoi figli, la sua paura per la nostra vita e la nostra salute, le lacrime che le avevano tracciato i solchi sulle guance, e la sua testa coperta di preoccupazioni con gelo grigio precoce. Ricordiamo le mani del padre, che non siamo abituati a guardare, ma dove conosciamo le tracce di fatica, tormento e cura che i figli e la vita possono avere in abbondanza. Le mani della madre e del padre sono le mani tese del Padre celeste, la sua dolce Provvidenza" (Wyszyński 2021, 178-179).

\subsection{I compiti connessi con la paternità}

La paternità è associata ai compiti connessi con la responsabilità della vita che nasce e viene affidata ai padri. Questo compito spetta non solo ai "padri della culla", ma anche ai sacerdoti, i padri spirituali. La paternità spirituale dei sacerdoti è un compito specifico che scaturisce dalla responsabilità nei confronti dei fedeli loro affidati, alimentando in loro la vita di Dio. Compito dei sacerdoti è essere "padre della santità e dei santi". Come implementarli? Il Primate del Millennio offre l'istruzioni specifiche. Esse rispecchiano un modello basato sull'attuale concetto di moralità. Molto prima dell'inizio del Concilio Vaticano II, Wyszyński incaricava i sacerdoti di seguire le sue raccomandazioni i quali assomigliano quelle esposte nel decreto conciliare un decennio dopo. Nel decreto conciliare sulla formazione sacerdotale si leggono intuizioni, che 
costituiscono le principali linee guida per l'esercizio della teologia morale: "Si ponga speciale cura nel perfezionare la teologia morale, in modo che la sua esposizione scientifica, più nutrita della dottrina della sacra Scrittura, illustri la grandezza della vocazione dei fedeli in Cristo e il loro obbligo di apportare frutto nella carità per la vita del mondo" (Concilio Vaticano II 1965b, 16).

Le raccomandazioni del Cardinale Wyszyński rappresentano la dimensione della moralità basata sulle virtù che rispecchia le idee dei padri del Concilio e sono molto attuali anche oggi. Il Primate raccomanda che la predicazione in chiesa non si concentri sulla descrizione dell'orrore del peccato, ma mostri la bellezza delle virtù. "In una parola, le energie vanno risparmiate per combattere il peccato, e da salvare per rappresentare la bellezza della virtù" (Wyszyński 2019b, 153). Questo non significa, ovviamente, che Wyszyński non vedesse le terribili conseguenze del peccato. Il testo dei Voti di Jasna Góra del Popolo Polacco, scritto durante l'isolamento del Primate, condannava numerosi vizi nazionali: pigrizia, avventatezza, spreco, ubriachezza, promiscuità. Un rimedio per esse sono le virtù morali che gli si oppongono: fedeltà, diligenza, laboriosità, rispetto reciproco, amore e giustizia sociale. Allo stesso tempo, il Primate stava preparando il programma della Grande Novena del Millennio del Cristianesimo in Polonia. Annotava poi: "La nostra debolezza e instabilità morale, nonostante la nostra fede forte, il nostro relativismo morale, incline a soccombere a cattivi esempi e correnti, obbedendo a vari errori, a volte semplicemente assurdi, alla caduta della moralità coniugale, all'infedeltà, alla licenziosità, all'ubriachezza, tutto questo fa sì che lo spirito sociale della nazione sia traballante. Sappiamo stare nelle chiese per ore, stare in piedi in piazza Jasna Góra come una vecchia quercia, ma soccombiamo facilmente anche all'eccitazione più debole per tutti i peccati e i vizi. Siamo spiritualmente raddoppiati, mentalmente distrutti, e quindi privi di uno stile di vita e di un carattere nazionale" (Wyszyński 2007, 119-120).

L'approccio del Primate alla teologia morale va comunque chiaramente nella direzione indicata più tardi dal Concilio Vaticano II. Sì, come osserva prof. Krzysztof Jeżyna, "i Padri conciliari non hanno preparato un trattato di teologia morale, quindi era necessario creare, quasi intuitivamente, un nuovo approccio a tutta la teologia morale, perché le linee guida dei documenti conciliari erano di carattere generale riguardo alla teologia in quanto tale" (Jeżyna 2015, 89). Lo studio dell'ascesi cristiana e l'insegnamento della vita nella grazia santificante devono essere di aiuto in tale annuncio della morale. In questo modo si può evitare uno degli errori più gravi della predicazione morale: concentrarsi sull'evitare il male a scapito del dovere di fare il bene. "Fac bonum - questo è un programma positivo. E a tale programma dobbiamo porre tutte le nostre energie - maestri di saggezza e santità cattolica - in ogni predica" (Wyszyński 2019b, 154). 
Nell'approccio conciliare alla teologia morale, è stato sottolineato molto chiaramente anche l'aspetto cristologico della vita morale. Lo ripetiamo: la vita morale si fonda sulla "grandezza della vocazione dei fedeli in Cristo". Giovanni Paolo II ha sviluppato questa idea definendo l'imitazione di Cristo come il fondamento originario e più profondo della morale cristiana (Giovanni Paolo II 1993, 19). Perciò le parole, le opere e i comandamenti di Gesù Cristo diventano la regola morale della vita cristiana (Giovanni Paolo II 1993, 21). Anche l'enciclica sul Vangelo della vita ha una profonda struttura cristologica. Giovanni Paolo II mostra Cristo in una prospettiva cosmica, come Buona Notizia per tutta l'umanità (cfr. Amato 1996, 127-144). Pure l'insegnamento morale del beato Stefan Wyszyński mostra chiaramente questo tratto cristologico. In una lettera ai sacerdoti scritta negli anni ' 50 , durante il suo isolamento forzato, definì lo scopo della vita umana come partecipazione alla vita di Dio. Possiamo raggiungere questo obiettivo solo in Cristo e per Cristo. La vita incentrata sul raggiungimento di questo obiettivo non avviene secondo il principio di "svelare il sipario per entrare nella scena di Dio e svolgere il compito assegnatoci nel meraviglioso spettacolo del mondo di Dio. Non siamo spettatori di come Zaccheo voleva che fosse. Siamo partecipanti attivi della vita di Dio" (Wyszyński 2019b, 63). Questa partecipazione attiva non si realizzerà se non "attraverso Cristo formando la propria personalità in unione, prima con Cristo e per Cristo con la Trinità. Perché ogni vita soprannaturale non è altro che partecipazione all'unità con la Santissima Trinità" (Wyszyński 2019b, 63).

\section{Conclusione}

Nel momento in cui viene scritto questo articolo, siamo poco tempo dopo la beatificazione del Primate del Millennio, il Cardinale Stefan Wyszyński. Il beato Stefan Wyszyński si unirò alla gloria degli altari che Giovanni Paolo II ha ormai raggiunto. Durante l'omelia di beatificazione pronunciata dal Cardinale Semeraro è apparso il filo del legame tra il Primate e il futuro Papa Polacco. Il Prefetto della Congregazione per i Santi ha ricordato le parole pronunciate dal Papa al Primate Wyszyński durante la prima udienza per i Polacchi dopo la sua elezione alla Sede di Pietro, confermando il ruolo del Primate nel preparare la strada al Karol Wojtyła al soglio pontificio (Semararo 2021). Il legame tra i due grandi Pastori della Chiesa Polacca e Chiesa universale esprimeva il senso della responsabilità per essere custode della verità del Vangelo, per il guidare il popolo di Dio. Ora si è realizzata loro comunità nella gloria dei santi del cielo. Entrambi grandi Pastori della Chiesa hanno mostrato la via della santità con le loro parole e con la loro vita. Adesso, per infallibile sentenza della Chiesa, ci presentano come raggiungerla. 


\title{
FATHER OF “BELOVED CHILDREN OF GOD” AND POPE OF THE FAMILY - TWO TEACHERS AND HERALDS OF THE GOSPEL OF LIFE
}

\begin{abstract}
SUMMARY
One of the important topics raised both by blessed Primate Stefan Wyszyński and in the teaching of St. John Paul II, there was the theme of life. The value of life includes both the temporal dimension and the supernatural participation of God's life in man. Primate Wyszyński and the Polish Pope consistently demanded respect for the inviolable nature of life from conception to natural death. God's grace in man determines the beautiful shape of life. Sensitivity to the beauty of the human soul is one of the important factors in the teaching of John Paul II, as well as characterizing the person of the Primate of the Millennium. The issue of life is also related to fertility and fatherhood. Fatherhood is expressed in the responsible fulfillment of tasks related both to the spiritual paternity of priests and to the physical paternity of fathers in the family.
\end{abstract}

Keywords: Wyszyński, John Paul II, life, fatherhood, love

\section{OJCIEC „UMIŁOWANYCH DZIECI BOŻYCH” I PAPIEŻ RODZINY - DWÓCH NAUCZYCIELI I GŁOSICIELI EWANGELII ŻYCIA}

\section{STRESZCZENIE}

Jednym $\mathrm{z}$ istotnych tematów podejmowanych zarówno przez błogosławionego Prymasa Stefana Wyszyńskiego, jak i w nauczaniu św. Jana Pawła II, był temat życia. Wartość życia obejmuje zarówno wymiar doczesny, jak i nadprzyrodzony udział życia Bożego w człowieku. Prymas Wyszyński i Papież Polak upominali się konsekwentnie o poszanowanie nienaruszalnego charakteru życia od poczęcia do naturalnej śmierci. O pięknym kształcie życia decyduje łaska Boża w człowieku. Wrażliwość na piękno duszy człowieka jest jednym z istotnych czynników nauczania Jana Pawła II, jak też charakteryzujących osobę Prymasa Tysiąclecia. Zagadnienie życia wiąże się także z płodnością i ojcostwem. Ojcostwo wyraża się w odpowiedzialnym wypełnianiu zadań związanych zarówno z ojcostwem duchowym kapłanów, jak też z ojcostwem fizycznym ojców w rodzinie.

Słowa kluczowe: Wyszyński, Jan Paweł II, życie, ojcostwo, miłość

Parole chiave: Wyszyński, Giovanni Paolo II, vita, paternità, amore 


\section{BIBLIOGRAFIA}

Amato, Angelo. 1996. Cristo, "il Vangelo della vita". W: Educare alla vita. Studii sul "Evangelium vitae" di Giovanni Paolo II, a cura di MarioToso, 127-144. Roma: LAS.

Benedetto XVI. 2006. Bóg darzy miłościa człowieka od chwili poczęcia. Przemówienie do uczestników Kongresu Papieskiej Akademii „Pro Vita” (Watykan, 27 lutego 2006 r.), L'Osservatore Romano, edizione polacca, 27 (6-7), 55-56.

Concilio Vaticano II. 1965a. Constituzione pastorale sulla Chiesa nel mondo contemporaneo "Gaudium et spes".

Concilio Vaticano II. 1965b. Decreto sulla formazione sacerdotale "Optatam totius".

Gatti, Guido. 1991. Morale matrimoniale e familiare. W: Corso do morale. III. Koinonia, a cura di Tullo Goffi-Giannino Piana, 391-399. Brescia: Queriniana.

Giovanni Paolo II. 1978. Nie byłoby tego papieża Polaka... Audiencja dla Polaków (Watykan, 23 października 1978 r.). Dostęp: 10.08.2021. https://www.vaticannews.va/pl/kosciol/news/ 2020-05/polska-wyszynski-nie-byloby-tego-papieza-polaka.html

Giovanni Paolo II. 1981. Esortazione "Familiaris consortio". Città del Vaticano.

Giovanni Paolo II. 1986. Wiara i kultura. Dokumenty, przemówienia i homilie. Rzym: Polski Instytut Kultury Chrześcijańskiej.

Giovanni Paolo II. 1988. Esortazione "Christifideles laici”. Città del Vaticano.

Giovanni Paolo II. 1991. „Nie zabijaj”: przeciw uzurpacji Bożej władzy nad życiem i śmiercia. Homilia podczas Mszy św. na lotnisku wojskowym w Radomiu (Radom, 4 czerwca 1991 r.), L'Osservatore Romano, edizione polacca, 12 (5), 34-36.

Giovanni Paolo II. 1993. Enciclica "Veritatis splendor". Città del Vaticano.

Giovanni Paolo II. 1995. Enciclica "Evagelium vitae". Città del Vaticano.

Giovanni Paolo II. 1997. Rodzina wspólnota życia i miłości. Homilia w Kaliszu (Kalisz, 4 czerwca 1997 r.), L'Osservatore Romano, edizione polacca, 18 (7), 35.

Giovanni Paolo II. 2007. Jaka przyszłość mamy budować? Przemówienie do Papieskiej Rady ds. Kultury (Watykan, 12 stycznia 1990 r.). W: Dzieła zebrane, V, 773-775. Kraków: Wydawnictwo M.

KAI. 2020. Dziś Narodowy Dzień Życia: Kard. Wyszyński w obronie dzieci poczętych. Dostęp: 05.12.2020.

https://www.ekai.pl/dzis-narodowy-dzien-zycia-kard-wyszynski-w-obronie-dzieci-poczetych/

Insegnamenti di Giovanni Paolo II. 1993. XV/2.

Jeżyna, Krzysztof. 2015. Odpowiedzialność Kościoła za dar zbawienia, Studia Theologica Varsaviensia UKSW, 53 (2), 89-105.

Listy Pasterskie Episkopatu Polski 1945-2000. 2003. Marki: Michalineum.

Lobato, Abelardo. 2003. Genitorialità. W: Lexicon. Termini ambigui e discussi su famiglia, vita e questioni etiche, a cura di Pontifico Consiglio per la Famiglia, 431-440. Bologna: EDB.

Merecki, Jarosław. 2019. Giovanni Paolo II - Wojtyła sulla sessualità. W: Dizionario su sesso, amore e fecondità, a cura di José Norriega, René \& Iabelle Ecochard, 433-435. Siena: Cantagalli.

Nagórny, Janusz. 1997. Między „kulturą śmierci” a „kulturą życia” - wyzwania współczesności. W: Evangelium vitae. Tekst i komentarze, red. Tadeusz Styczeń, Janusz Nagórny, 135-158. Lublin: RW KUL.

Rodziński, Adam. 1998. L'amore alla base del costituirsi della cultura. W: Na orbitach wartości, red. Adam Rodziński, 201-214. Lublin: RW KUL.

Semeraro, Marcello. 2021. Homilia wygłoszona podczas beatyfikacji ks. kard. Stefana Wyszyńskiego i Elżbiety Róży Czackiej (Warszawa, 12 września 2021 r.). Dostęp: 14.09.2021. https://www. radiomaryja.pl/kosciol/homilia-ks-kard-marcello-semeraro-wygloszona-podczas-beatyfikacjiks-kard-stefana-wyszynskiego-i-elzbiety-rozy-czackiej

Wojtkun, Jarosław. 1998. Il valore dottrinale dell' "Veritatis splendor" e dell' "Evangelium vitae". Roma. 
Wojtkun, Jarosław. 2020. Rodzina w stużbie życiu i miłości. Radom: Spatium. Wyszyński, Stefan. 2007. Wszystko postawitem na Maryję. Warszawa: Soli Deo. Wyszyński, Stefan. 2017. Pro Memoria, t. 1: 1948-1952. Warszawa: IPN. Wyszyński, Stefan. 2019a. Pro memoria, t. 8: 1961. Warszawa: IPN.

Wyszyński, Stefan. 2019b. List do moich kapłanów. Warszawa: Soli Deo.

Wyszyński, Stefan. 2020. O godności kobiety. Zielonka: Sumus.

Wyszyński, Stefan. 2021. Wiara i odpowiedzialność. Zielonka: Sumus.

Jarosław Wojtkun - kapłan, doktor nauk teologicznych, Katedra Teologii Moralnej Społecznej Wydziału Teologii KUL, adres: wojtkun@kul.lublin.pl 